\title{
Osservazioni limnografiche sul lago di Albano (*)
}

\author{
P. CALOL - G. ROMUALII
}

Riceruto il $1^{\circ}$ Novembre 1964

\begin{abstract}
Riasento. - Si riassumono i risultati di alcune serie di registrazioni himnografiche, eseguite in epoche diverse sul lago di Albano, allo scopo di confrontare le caratteristiche delle uscillazioni libere del laro, calcolate per via teorica, con quelle reali.

E risultato, come fer altri lashi italiani, un ottimo atecordo tra teoria ed usservazione, per quanto concerne i periodi e gli spostannenti relativi ai due estremi. Conformemente alla teoria di Hidaka, risulta cusì che lampiezza dell'uninodale all'estremo Nord del lago (riva abitata) è circa quattro volte quelia che si registra rulla riva opposta.

Cna particolarita del lago di Abano - the risulta, sostanzialmente, dalla fusione di due crateri, quello settentrionale in parte franato nel più profondo, meridionale - - fa si che la parte a settentrione si comporti come golfo aperto sul resto del laggo. Pojiché il periocio naturale di questa parte del bacino is dell'ordine del periodo dell'uninonale dell'intero lakro, molto spesso si registrano, sulla riva is settentrione. oscillazioni di circa $4^{\mathrm{m}}$, peculiari dei bacini aperti, in se conchiuse, e la eni ripetizione ì strettamente legata al persistere della causa perturbante. del lago.

Da ultimo, si valutano i valori medi dell'energia, associatat alle uninodali
\end{abstract}

SUMmary. - The paper reports the results of a series of limnographic surveys, carried out at difierent times wn the lake of Albano, in order to compare the eharacteristics of free oscillations of the lake, calculated theoretically, with the real ones.

The results have shown, as in the case of other Italian lakes, a very good agreement between theory and observation, both as to the poriods and as to the range between the two extremes. In conformity with Hidaka's theory, the uninodal amplitude at the Northen extreme of the lake (inhabited side) appears to be four times lighers than that which is noticed on the opposite side.

$\left(^{*}\right)$ Questa ricerea è siata condotta con contributi del Consiglio Nazionale delle Ricerche. 
A particular feature of the lake of Albano -.. which is composed, basirally, by the union of tho araters; the Forthern one, partly sledged into the fouthern one, whith is deeper... is such that the Northern seetion behaves like a gulf open on the rest of the lake. Nince the natural period of this side of basin is in the range of the uninodal period of the whole lake. wh the Vorthern side it is often prosibile to record uscillations of about $4^{\mathrm{m}}$, typical of open basins. The repetition of these oscillations is strietly connected to the continued presence of the catlise of pertubation.

Finally, the paper evaluates the average values of the energy associated with the lake's uninodal.

Te atratteristiche fondamentali delle ose illazioni libere del lago di

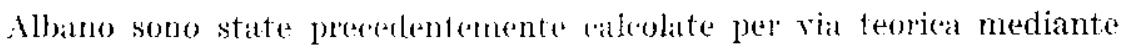
i metodi di befant, Hirlakit a Chrystal da uno di noi esposti, con opportune esterisioni te correzioni, nel $1948\left({ }^{(1)}\right.$; nella tabella seguente sono riassunti i dati relativi at jeriodi e ai nodi (2, 3).

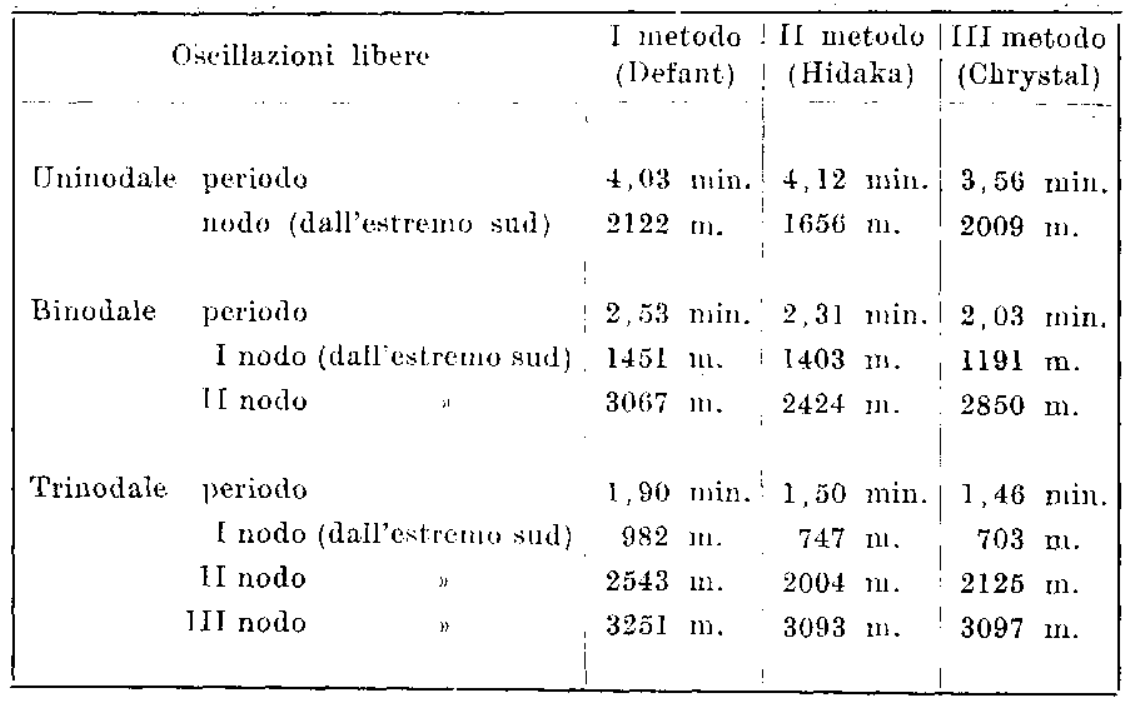

I tre metodi hanno permesso di studiare l'andamento delle ampiezze in senso verticale lungo l'asse maggiore del lago. $\mathrm{E}$ risultato in partirolare che all'estremo Nord, in corrispondenza cioè della parte meno profondit del lagro, le ampiezze verticali devono essere sensibilmente maggiori che nella parte meridionale, dove si riscontra la massima profonditic. Tale caratteristica è stata riscontrata per tutti e tre i tipi d'o- 
scillazione e con tutti e tre i metodi, $\mathrm{i}$ quali rutlatia differiscono in quel che riguarda l'entità degli spostamenti rerticali relativi.

Allo scopo di verificare la validità dei risultati teorici, fra il $195 \%$ e il 1963 sono state compiute drelle serie d'osservazioni sul posto. Durante tutti i periodi d'osservazione un limnografo ha funzionato nella parte settentrionale del lago; contemporameanente nel 1957 sono state effettuate alcune registrazioni in località prossima all'uninodo e nel 1963 nella parte meridionale del lago.

ESPERLENEe FSEGLITE Fra IL 12 E IL 2.1 APRLE 1957.

Le registrazioni iniziano il 12 Aprile 1957 a proseguono ad intervalli più o meno lunghi fino al $2+$ Aprile.

Un primo periodo di recistrazione inizia il 12 Aprile verso le 18 e prosegue fino alle 22,30 dello stesso giomo. In tale intervallo di tempo vengono registrate ampie oscillazioni uninodali $\left(T=4^{\mathrm{m}}, 16\right)$ alterate da altre oscillazioni e da perturbazioni (Fig. 1). Si può fra l'altro notare che l'uninodale sembra ricevere di quando in quando un impulso più forte, che determina oscillazioni sensibilmente più ampie. Tale fenomeno appare più whiaro fra li’19 e le 21.

Tale fenomeno is dovuto -... eme velhemo a. al contemporanco insorgere di sesse da zoccolo, ha cui uninodale presenta un periodo pressochè uguale al periodo dell'uninodale dell'intero lago.

Alla ripresat della registrazione verso le 9 del 13 , l'uninodale ( $T=4,12 \div 4,16$ minuti) presenta un'ampiezar nottamente inferiore (Fig. 1a), mentre appaiono atlenuate, ma sempre presenti, le coperiodali sesse da zoccolo.

La registrazione viene interetta alle 19,15 del 13 , senza che nel frattempo si siano avute oseillazioni diverse dall'uninodale.

L'esistenza pressochè esclusira di oscillazioni uninodali, con periodo sui $4,10 \div 4,12$ min., cui si sovrappongono talvolta "shelf-seiches", earatterizza il periodo di registrazione fra le 9,20 del 14 e le 9,30 del 15 (Fig. 1b). La ripresa della registrazione alle 11,45 del 15 mette in evidenza un complicarsi delle oscillazioni del layo. Tale agitazione prosegue accentuata fin verso le 20 del 16 , quando cominciano a prevalere le oscillazioni uninodali, sovrapposte a binodali e trinodali.

Le uninodali presentano però, spiecata la caratteristica propria delle oscillazioni proprie dei bacini aperti (o limitati da zoccolo), che si susseguono l'una all'altra, come impulsi separati (Fig. 2). 
Le uninodali (sempne prò disturbate) prevaluono volso le nove dral 17 .

Varso le 13,15 l'uninodale romincia ad essere bordata da "shelfsciches" $\left(T \cong 1^{\mathrm{m}}\right)$; suceressivmente allosedlazione fondamentale si sovrappengono deboli binodali $(2 \mathrm{~m}, 3)$ o 1 rinodali $\left(1^{\mathrm{m}}, \bar{i}\right)$.

Dalle: $18^{\text {b }}$ alle $21^{\text {b }}$ (Fig. 3) si ha tulto un seguito di singole uninodali fa zocoolo separate da intervalli di qualche minuto, mas dallaltra. Ĺuninodale dal lagro si presenta alla ripresa delle regist maioni alle 9,30 del 18. L'ampiozza va prós diminuendo, mentro intorno alle 11 inizia

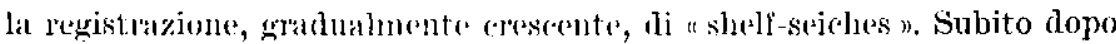
all'uninodale si alternano anch oscillazioni trinodali $\left(T \cong 1^{\mathrm{m}}, 7\right)$ e binodali $\left(T \cong 2^{m}, 1\right)$; quest altime appaiono molto chiare far le 18 e le 20, dopo di the si hat ma serie (non molto accentuata) di oscillazioni uninodali alternate a binordali fino alle 23,45 . Ta registrazione riprende,

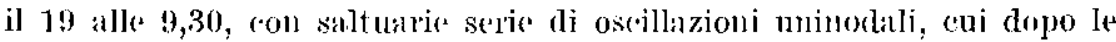

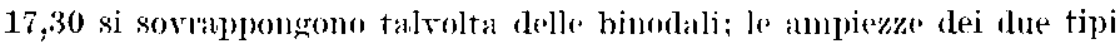
di osrillazione' sono molto ridotie.

Il sriol'no 20, dopo le 13 all'uninodale si alter'batno altre oscillazioni con periodi inferiori al 2 minuti; dopo le 16 insorgono oscillazioni

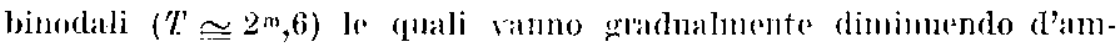
piezza fino a scomparire netle prime are del 21. Fiar le 9 a le 10 di tale yiorno, inizia un periodo di anitazione "he si areentua verso le $10,30$. Fia le value oscillazioni disordinate si notano saltuariamente delle serie di oscillazioni a periodo di cir'at lm,70 (trinodali), eni verso le 15 segruono oscillazioni più lente $\left(T \cong 2^{m}, 40\right.$ binotali) ('lne vinno gradual-

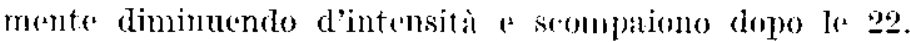

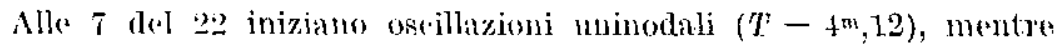
piì tardi velso lo 1.2 sexper un nuovo prepiodo d'agitazione con origine di trinodali molto chiate $\left(T^{2}=\mathrm{I}^{\mathrm{tn}}, 70\right)$, sovalpeste a oscillazioni uni-

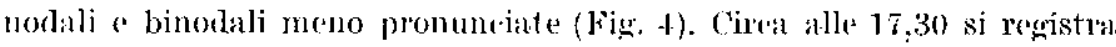
un improviso abbassamento del livello del lago (Fig. 4a) provocato dal pasiaggio di un disturlo mirobarieo positivo. La registrazione termina alle 19,15 .

L'apparecelhio riprende il funzionamento il giorno 23 alle 8,30 . Dopo alcuni aternni di uninodali, eui peraltro si sovrappongono oscillazioni a più brece periodo (cirral 1 min.), la registrazione si compliea sia per l'areentuarsi di tali oscillazioni sia per la presenzat saltuaria di esempi di rinodali (velso le 12,$\left.15 ; T \cong 1^{m}, 75 \div 1^{m}, 70\right)$ o di binodali. Ite oscillazioni binodali vanno poi grardualmente prevalendo; dalle 18 in poi si ha infati la legistrazione pressochè eschusiva di tali oscillazioni 
che mostrano un periodo medio di $2^{m}, 72$ (Fig. is a $5 a$ ). Ferso lo due rel 24, la binodale comineia at essere bordata da oscillazioni a periorlo intorno all minuto.

Purtroppo dalle 5 del 24 fino alle 15,50 , wioc tino al termine della

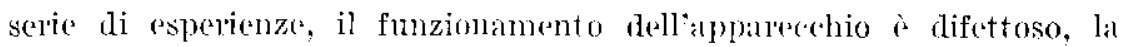
registrazione si hat solo per brevissimi tratti.

Nei giorni 18, 19, 20 a $23-24$, oltre alle lentistrazioni del limnografo situato nella parte setfentrimale da lago, si hamo le registrazioni di un secondo apparerehio situato in località prossima all'uninorlo.

Nel periodo dal 18 al 20 , in tale localiti non si leogistral alema uni-

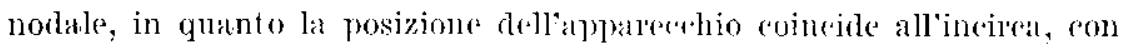
l'uninodo. Si hatmo solo vibrazioni per veluto a qualche traceia di binodale.

Per la registrazione fra il 23 a il 24 , rispetto alla registratione del primo apparecchio si puo notare che, restando irdentiche le caratteristiche generali della regristrazione rhe inizia alle 9,15 quando si ha già la presenza di brevi oscillazioni forzatra, diminuisce lampiezza della binodale a della trinodale, mente si accentuano le oscillazioni a più breve periodo.

In ogni modo, come era da attendersi, mancano totalmente le uninodali.

LSPERTENZE ESEGLITE NEI 1958.

Due nuove serie di registrazioni limitate all'estremo Nord del lago, furono eseguite rispettivamente nel Harzo e nel Novembre 1958.

Il 10 Marzo 1958 la registrazione, per quanto saltuaria, sorprende l'insorgere di una serie di oscillazioni minotali di ampiezza eccezionale (Fig. 6). La registrazione anpare analoga a quella del 12 Aprile 1957. Anche qui l'uninodale appace turbata da altre oscillazioni anch'esse di notevole ampiezzi. Tali impulsi supplementali spariscono man mano ohe l'ampiezza dell'oscillazione fondamentale dintinuise's. Verso le ore 18 si può infatti osservare come l'uninorale si normalizzi con l'improrviso diminuire dell'ampiezza. Fino a dopo le 0,30 dell'11 Mal'zo si ha una serie ininterrotta di oscillazioni uninodali (Fig. 7) in cui appare evidente come al decrescere di esterni fatori perturbanti la forma dell'oscillazione si perfeziona con il diminuile dell'ampiezza.

Di pari passo con tale attenuazione dell'onda sembra esservi un aumento del periolo ohe va dai 3,96 minuti ai 4,15 minuti. 
Verso lo 0,45 dell'11 l'uninodale appare bordata da "sluelf-seiches" mentre l'ampiezza rellonda comineia ad aumentare.

Purtroppo la registrazione va fuori "arta poro ropo le 2. Rimessa a posto verso le 7,20 mostra dapprima una fitta seje di oscillazioni a periodo inferiore al minuto (vibrazioni per vento "rapidi disturbi microbarici) su cui prevale gradualmente loscillazione uninodale ad ampiezza molto clevata $\left(T=1^{m}, 19\right)$. Ja remistriziont continua ad essere saltuaria e cessal verso le 14,30 .

L'apparechio ripende a funzionare il 13 alle 13,30. La registarazione inizia con una serie di uha ose la eui ampiezza aumenta improvisamente a rapidamente fra le 150 le 15,30. Contemporaneamentr, alluninodale, si sovappone un'oscillazione binodale ohe appare molto (hiama (Fig. s) verso le 16,30 quando diminuise l'ampiezza dell'uninodile. Fra le 16,30 e le 17,15 la binodale $(T-2 \mathrm{~m}, 18)$ ha il sopravento sull'uninodale cui torna poi a sovrapporsi. Dopo le 21 è presente solo l'oscillazione uninodale (Fig. 9) che con :mpiezze vare, viene registrata fno verso le 22 del 16. Poi va gradualmente scomparendo e fino alle 12 del 15 , ora in cui termina la registrazione, non si ha quasi nulla; solo saltuariamente si notano delle deboli uninodali.

La registrazione riprende alle 11,34 del $16 \mathrm{~mm}$ fin verso le 21

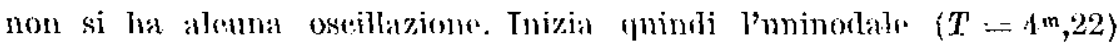
bordata suecessivamente dit "shelf-sedetes"s; amberdue i tipi di oscillazioni hanno ampiezze crescenti (Fim. 10). Verso le 0,3 del 17, all'uninodale si sovarppone la trinodale $\left(T \simeq 1^{\mathrm{m}}, \overline{7}\right)$.

Lat registrazione termina il 17 Aprile verso le 11.

La seconda serie di registrazioni del 1958 inizia il 26 Novembre alle 16,38 .

Fino alle 20 dol 26 non si registra alcuna oscillazione particolare, poi si ha ma serie di osrillazioni forzate cui segue una lunga serie di oscillazioni uninodali $\left(T \cong t^{\mathrm{m}}, 36\right)$. lalvolta bordate da "shelf-seiches " e talvolta molto attenuate.

Dalle 13,30 del 27 si ha un lungo periodo con esempi rari e attenuati di uninodali.

L'uninodale si rinforza verso le 01 del 28 . Verso le 03 l'uninodale comincia ad essere turbata da ampie oscillazioni forzate (Fig. 11), che si attenuano fra le 8 e te 9 per poi riprendere con rinnovata intensità, fino a circa le 17. In questo periodo l'oscillazione forzata oltre ar essere sovrapposta all'uninodale appare talvolta sovrapposta alla trinodale $\left(T \cong 1^{\mathrm{m}}, 8\right)$ a alla binodale $\left(T \cong 2^{\mathrm{m}}, 6\right)$. 
Di scarso interesse risulta il periodo rli registmaione che va dalle 16,10 del 30 Novembre alle 16,40 del 10 Diembre: infatti solo in alenui momenti si la la registrazione di un'uninodale molto attenuata.

ESPERTIENTI EFFETTLATI DAL 60 M 13 MLARZO 1963.

Le registrazioni effettuate nel Aarzo 1963, averano come scope di verificare quanto previsto dalla teoria sullandamento delle ampiezze.

Furono pertanto effertuate contenporaneamente due serie di registrazioni una all'estremo Nord e ma allestremo Sud.

Nella parte Nord la registrazione inizia verso le 17 del 6 Marzo mentre nella parte Sud inizia virso le 9,30 del $\bar{\tau}$. lino a verso le 10,30 del 9 non si registra però aleuna oscillazione.

A tale ora inizia a Ford un'uninodale molto (hiara $\left(T=4^{m}, 06\right)$ che a Sud appare appena aceennata.

La registrazione sureessivamente diventa incerta anche perché il limnografo è difettoso a caluxia di forti venti. En confronto può essere fatto nuovamente pere le le resistazioni compresse fra le 16,29 del 10 Marzo e 8,30 dell'11 e fra le 10 e le 13 detl’11 (Figg. 12, 13 e 14).

Si può notare eome l'uninotale regristrata ron estrema chiarezza $\left(T=4^{\mathrm{m}}, 06\right.$ ) abbia nella parte meridionale del lago (Figge. $13 a$ e 14a) ampiezze inferiori anche di 93 risecto alla parte settentrionale (Firy. $13+14)$.

Fra le 11 e le 1.3 dell 1.1 . Marzo 1963 all'uninodale cominciano a sovrapporsi oscillazioni clovute al vento che turbano la registrazione. Inoltre nella parte setentrionale la registratome si interompe alle 13 , per cui è impossibila un ronfronto.

Nella parte meridiomale drel lago dove rontinua la registrazione fin dopo le 05 del 12. nette oscillazioni dovute al vento continuano a sovrapporsi all'uninodale e in alcuni asi alla binodalt.

Un confronfo fra oscillazioni della parte meridionale e della parte settentrionale è di nuovo possibile fra le 10 del 12 Marzo e le 12 del 13.

Dalle registrazioni di questo intervallo di tempo si può notare come non solo l'ampiezza dell'uninodale ma anele quella delle altre oscillazioni sia nettamente superiore nella parte settentrionale del lago.

L'inizio delle rexistrazioni coglie il lago. in ma serie complessa di oscillazioni: infatti, all'uninodale $\left(T=4^{n}, 05\right)$ aplaiono sovrapposte oscillazioni binodali $\left(T=2^{m} \div 2^{m}, 5\right)$ e trinoxlali $\left(T \cong 1^{m}, 7\right)$, che nolla parte seltentrionale del layo hamo ampiezze molto aecentuate. 
Il massimo delle ampiezze i ha fra le 13 e le 15 (Fig. 15); poi si ha contemporaneamente un graduale dimimuire delle ampiezze e il prevalere de Il'oseillazione' uninodale.

Je oscillazioni binorlali a trinodali seompaiono verso le 18 nella parte meridionale de] litgo a verso le 22,30 mella parte setientrionale:

Sempre a causa della minole ampiezza d'oscillazione, l'uninodale, whe nella parte settentrionale continua ad essere registrata ininterrottamente dopo le 22,30 fino alla line della rexistrazione, nella parte meridionale del lago scompare pressochè definitivamente già verso le. 21 .

\section{CONCLUSTONI.}

I tre periodi dosservazione sopra riassunti, lamno consentito di trare aleune conchusioni sullidtodinamia del lago d'Albano (o di Castelgandolfo, ehe dir si voglia).

Come avviene per la generalita dei laghi italiani, finora investigati, anche nel lage di Albano l'oseillazione libera predominante, per ampiezza o frequenza, è la fondamentale (uninodale).

A ditterenza di altri laghi italinni, di analoghe dimensioni, le oscillazioni libere binodali e trinodali si presentamo pure di sovente, talora ron notevole ampiezza.

Anche il lago di Albano à spesso commosso da lunghe serie di "shelf-seiches" (sesse da zoc'olo), con periodi dell'ordine di un minuto, generahmente associate a forti venti o al transito di rapidi disturbi microbitrici.

A proposito delle sesse da zoreolo, va fatta un'imjortante precisazione per quanto conererne il lago di Albamo. Una semplice osservaziones della sezione longitudinale, centrale, del lago, mostral che esso risulta dalla congiunzione di due bateini ( $(\cdot a t+r i)$, il più profondo costituendo la parte meridionale del lago. 11 bacino settentrionale, più basso, scende a dolee declivio verso quello profondo, che raggiunge praticamente verso lisobata 40, in corrispondenza della quale il fondo precipita. Il bacino settentrionale, quindi, può ritenersi come un golfo aperto sulla restante parte del lasgo. Pertanto, se il periodo dell'uninodale risulta inferiore od uguale a quello della fondamentale dell'intero lago, le condizioni fisiche per la sua libera eccitazione sarebbero rispettate. E quanto efcettivamente si verifica. 
Il valore applossimativo del periodo del bacino settentrionale. considerato come golfo, può ottenersi applicando la nota formulal.

$$
T_{s e t}=\frac{1 l}{\sqrt{g h}}
$$

dove l esprime, nel nostro caso, la lunghezza del golfo in parola (contata sulla linea di valle) e $h$ la protondità media lungo tale linea, essendo $g$ l'aecelerazione di gravita. Dalla sezione longitudinale del lago e dai dati batimetrici, si ottiene

$$
4 l=2880 \mathrm{~m}, \quad h=15 \mathrm{~m} .
$$

L'applicazione della formula conduce allora al valore approssimativo

$$
T_{\text {snt. }}=210^{\mathrm{s}}=4^{\mathrm{m}} \text {. }
$$

Il periodo fondamentale della parte meno profonda del lago ha quindi un valore di fure cires, dell'ordine cioc dell'uninodale dell'intero lagro.

E questa ma peculiale particolarità del lagro d'Albano, che consente la chiara spienazione di fatti altrimenti inspiegabili. Sovente oscillazioni con periodo di 4 minuti cil'a si presentano come impulsi isolati, in se conelusi, separati da brevi intervalli oceupati da altri tipi di onde; ciò si verifiem sperialmente culundo l'ampirzac dell'onda è molto accentuata (Fig. 1), mat avriene and he - sia pure memo frequentemente per piecole ampiezze (Fig. 2). E noto che oscillazioni del genere sono proplie dei barini auerti: formatasi l'ondir propria del bacino (che ha il suo nodo in corrispondenza dell aperetura del golfo), oltre il nodo l'onda stazionaria si trasforma in onda progeressiva verso il larego. Ta formazione di una nuova oscillizione libelat richiede il persistege della causa pert ulbante: più questa dulal, più numerosa sirà la serie delle singole onde separate. E cosi ohe si spiesa, dal punto di vista irlrodinamico, l'aceennato fenomeno, "alitferistieo dre liseo di Albano.

Quando il mereanismo della callsa perturbante i tale da interessiare l'intero lago, avremo te oscillazioni libere di tutto il bacino: uninodali, binodali, trinodali,... In questo ciso, l'uninodale si susseguirà in lunghe serie senza soluzione di continuità da un'onda alla successivis (Fig. 12): la loro durata è Iexata al coefficiente di smotzamento naturale delle oscillazioni libere, a meno che, nel frattempo, non intervengano move cause di perturbazione.

So ora passiamo a confrontare i dati dell'osservazione con quelli ottenuti per via teorica, notiamo che - almeno per quanto concerne 
i periodi predominanti -- il meterle che las condotto ai valori più prossimi a quelli osservati is il metodu di Hidaka (2). Ancles il motodo di Defant ha fornito valori per i periodi in buon aceordo con la media di quelli rati. Il metordo di Chrystal $\left(^{3}\right)$ porta a valori leggermente infel'jori alla media. La schelnatizzazione della rurva normale che tale metodo comporta, talvolta si liflutte apprezzabilmente sulla attendibilità dei risultati: nel aso in esime, laz concotto a periodi lievemente inferiori a quelli ot temuti diallosservazione.

Per quanto concerte i rapporti delle ampiezze delle uninodali dell'intero lage, ai suoi estremi, l'osservazione mostat, in merlia (nei brevi periodi in eni is stato possibile regist rate contemporaneamente ai due estremi), rhe l'uninodale ral lato sattentrionale raggiunge ampiezze circa 3,8 volte quelle laswiunte rontemporaneamente al lato opposto. bei metodi applixati, quello di Defant dà un lapporto dello stesso ordine

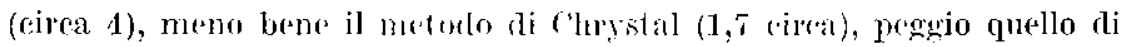
Hirlaka.

Restano ancora da rerifirare lo posizioni dei nodi e gli spostamenti orizzontali rhe le particelle liquide subiseono in essi, quali risultano dai metodi applicati. Questa parte viene rimandata ad ulteriori indagini.

Fin d'ora, possiamo invece oftenere -... sulla base delle legistrazioni escugute -.. un valore approssimativo dell'encugia associata ad un'oscillazione uninodale di ampiezzal noedio-massima (fra quelle registrate).

Equiparando Palveo di un lago ad un bacino di forna parallelepipeda, di lunghezza $l$, larghezza $b$ e profondità $h$, si prova $\left({ }^{4}\right)$ che l'energia cinetica $E_{c}$, associata ad un'oscillazione libera minodale di periodo $T_{o}$, assume l'espressione"

$$
E_{c}-\frac{1}{2} \rho b h l C^{2} \sin ^{2} \frac{2 \pi}{T_{0}} t
$$

essendo $\varrho$ la densità dell'acequa e.

$$
C=H \quad \frac{g}{h},
$$

dove $H$ rappresenta l'ampiezzal dell'uninodale considerata.

Dalle colonne $3^{\mathrm{a}}$ e $4^{\mathrm{a}}$ della Tabella I del primo lavoro sul lago d'Albano $\left({ }^{2}\right)$, dove sono riportate le superficie delle sezioni trasversali e delle loro larghezze in superficic, è facile dedurre le profondità medie delle sezioni stesse, equiparate a rettangoli; la media di tali profondità, darà la profondità media $h$ del lago, mentre la media dei valori della su detta 
1a colonna fornirà $b ; l$ è il valore riportato, come ultimo, nella $2^{a}$ eolonna della stessa Tabella. Per il lago di Abano si ottiene pertanto

$$
h:=63,7 \mathrm{~m} ; \quad b=1590 \mathrm{~m}: \quad l:-3575 \mathrm{~m} \text {. }
$$

Fitto $H=\breve{s}$ cm., ronstegule

$$
(2-3,8325) \text { ('m11/sece. }
$$

Per $Q=1 . \mathrm{gr}^{2} / \mathrm{cm}^{3}$, si ottiche infine

$$
\begin{aligned}
E_{c} & -3,5.10^{14} \text { trgs } \\
& -9,7 \mathrm{KWh} .
\end{aligned}
$$

Il valore medio dell'energia associata ad uninodale dell'ampiozza di $5 \mathrm{~cm}$. ̀ quindi, per il lago di Albano, dell'ordinc di $10 \mathrm{KWh}$. Poiche nel lato meridionale del lago, l'ampiezza dell'minodale è circa $1 / 4$ dei valori che essal assume sul lato opposto, possiamo conchadere che la corrispondente euergiat ì rirca $1: 16$ di quella ivi sviluppata.

\section{BIBIOLORABLA}

(1) Canor l'., Le sesse del lago di Garda. Parte l o Parte ll. "Annali dj Geo. fisica $n$, I, $1,2(1948)$.

(") Gronar M., Studio sulle sesse del lago di Albano. "Annali di Geofisica", I, 4 (1948).

(') Car.or P. \& Gromar M., sulle oscillazioni libere del lago di Albano. "Allnali di Geofisica ", V, 2 (1952).

(4) Calor P., Oscillazioni libere del lago di Bracciano; loro caratleristiche $e$ loro energia. "Annali di Geotisica ", XI, I (1958). 


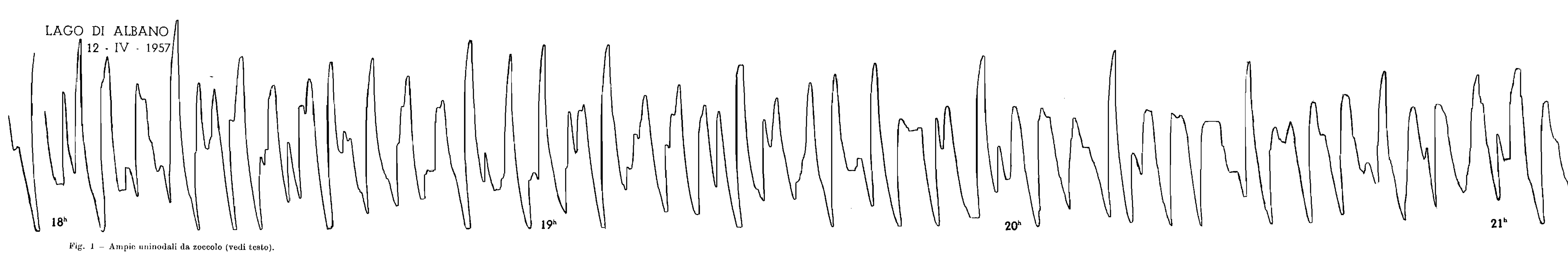

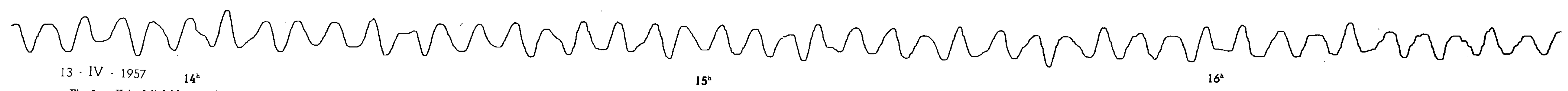

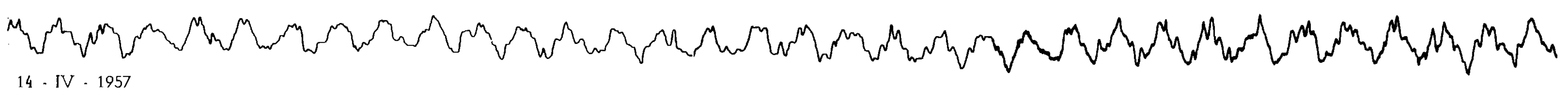
$14 \cdot \mathrm{IV} \cdot 1957$ 


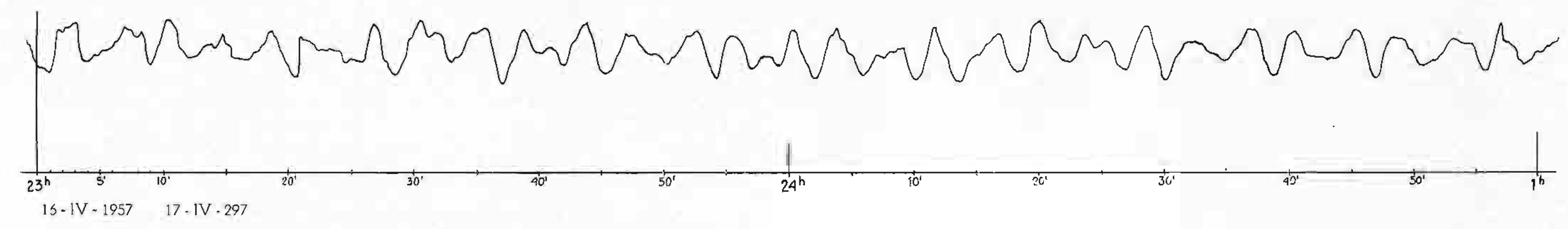

Fig. 2 - Esempi di nainodali da zoceolo: si noti la successione di oscillazioni singole isolate.

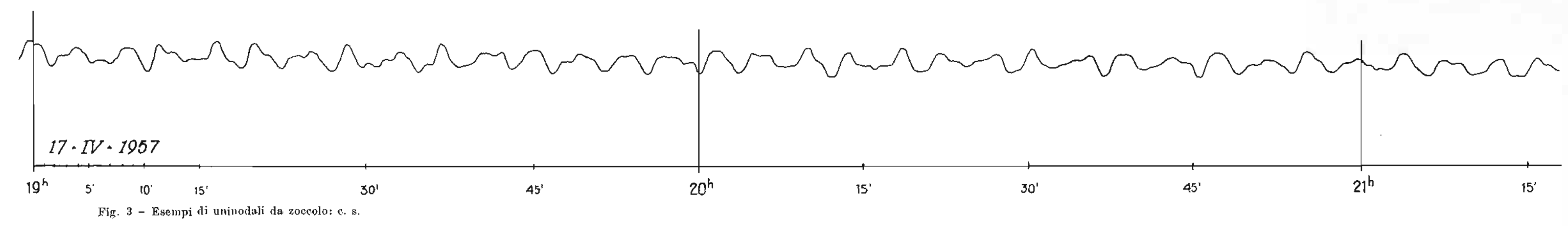




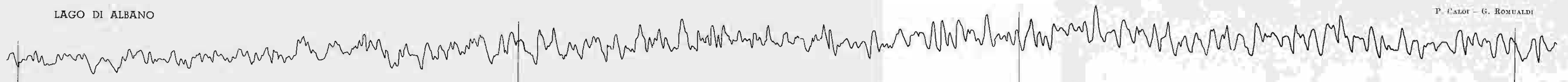
$22 \cdot \pi \cdot 1957$
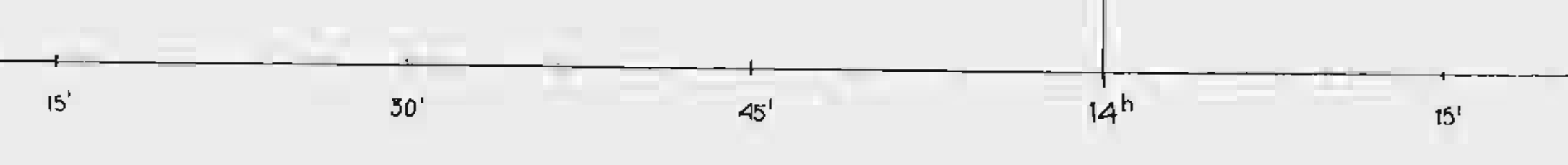

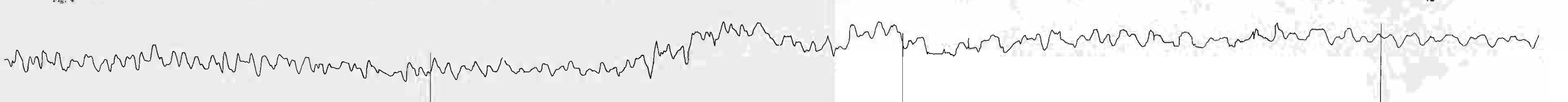

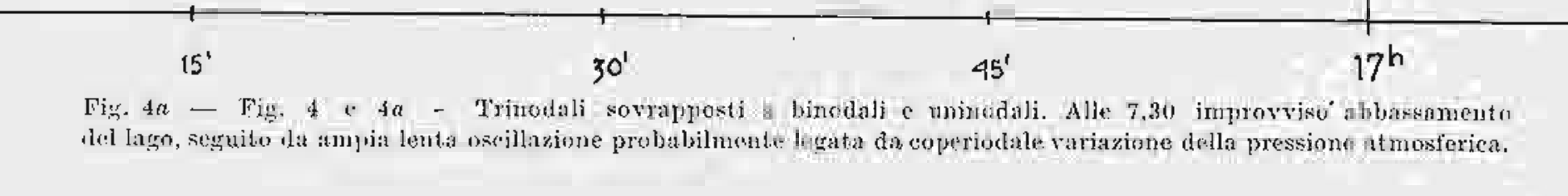

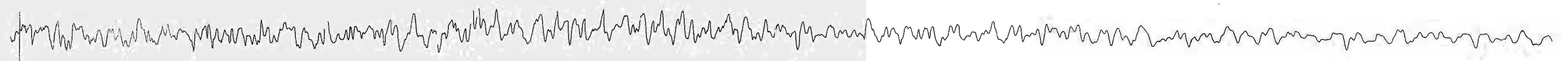
$23 \cdot \pi V \cdot 1957$

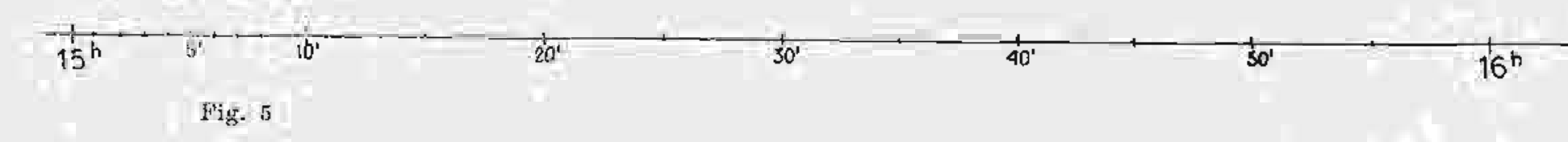

moromangrum 


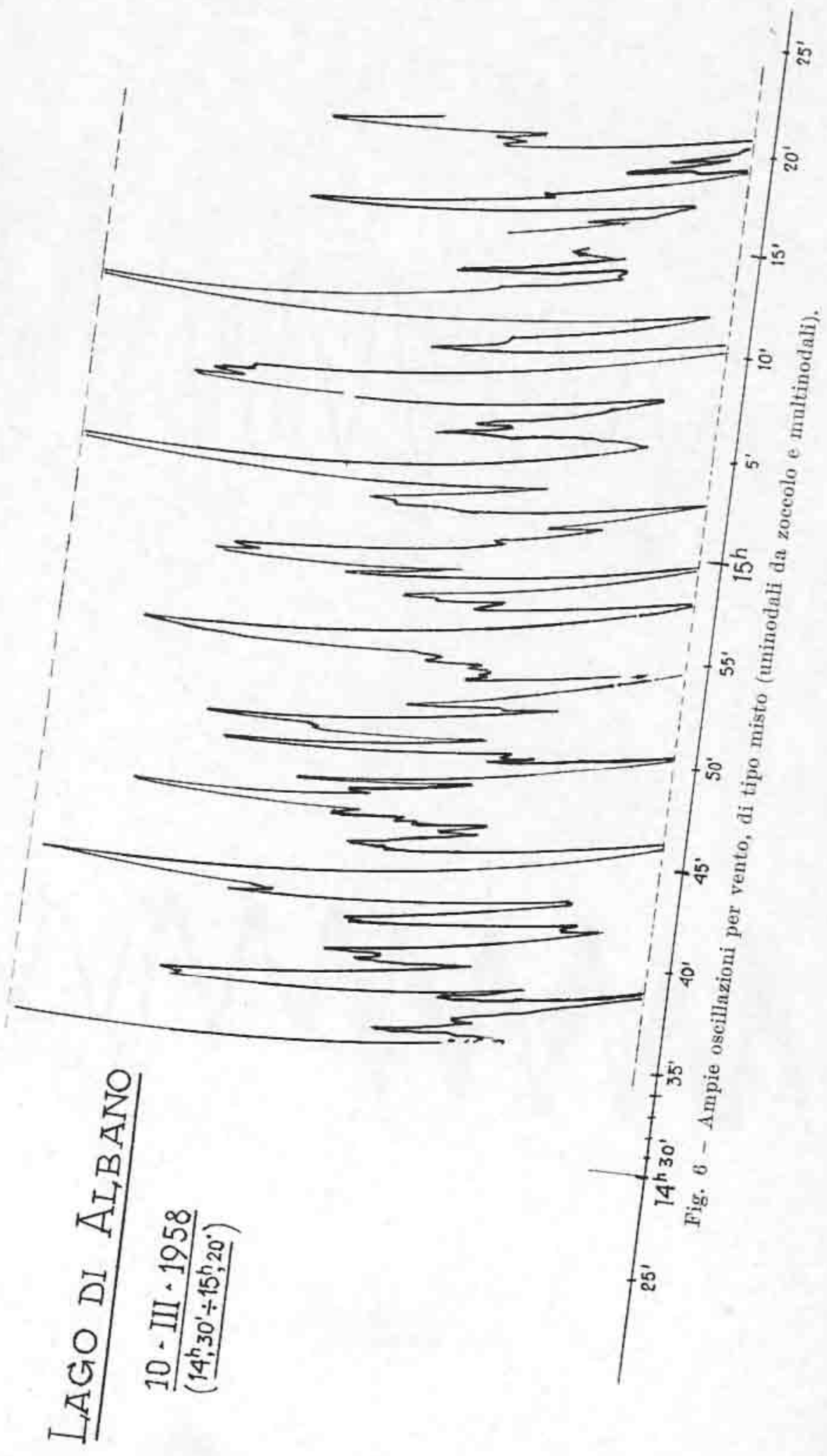




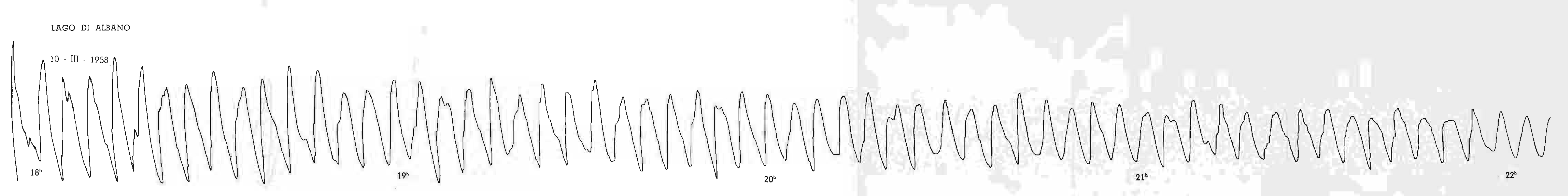

mon-monnm

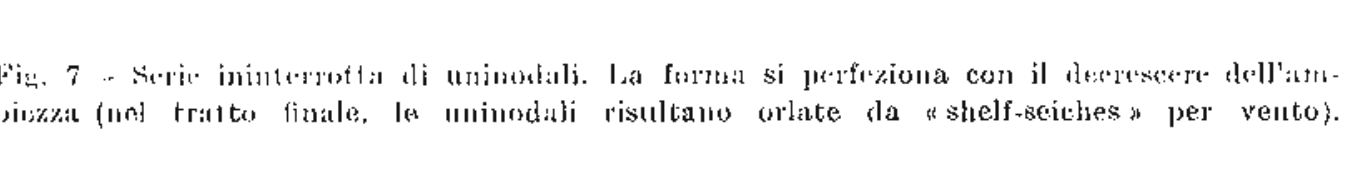




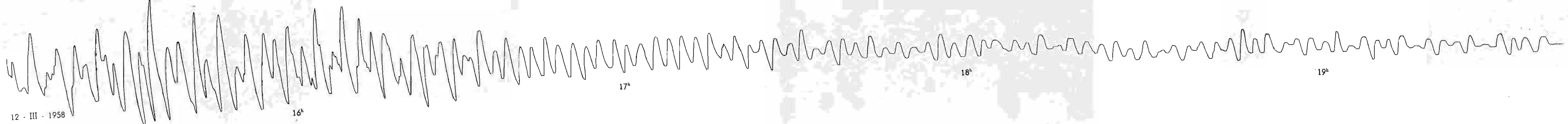
12. III. 1958 .

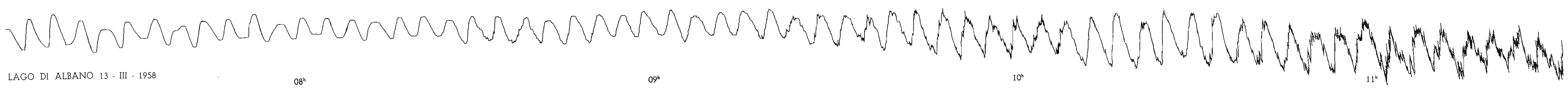




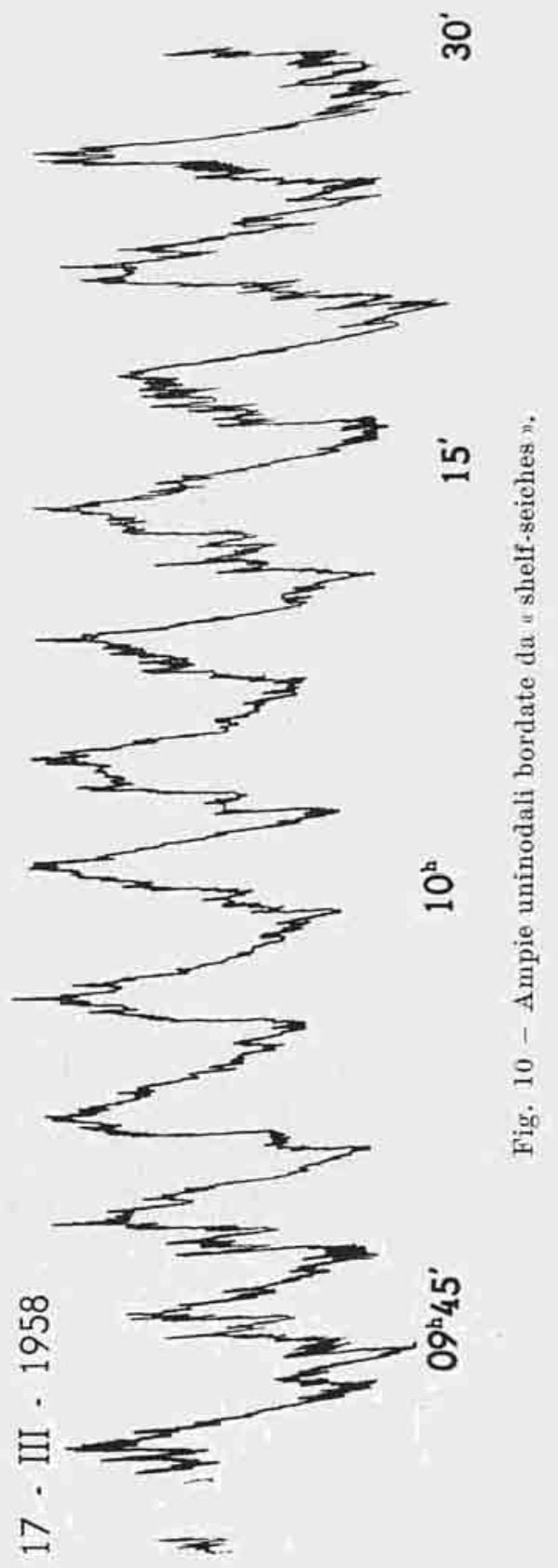




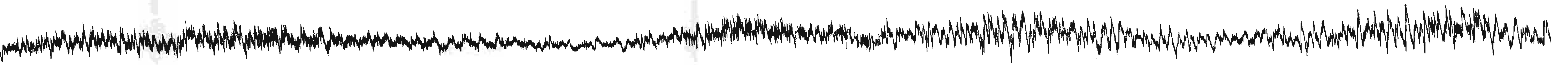

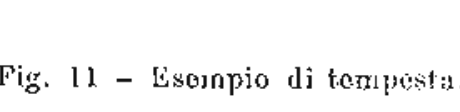




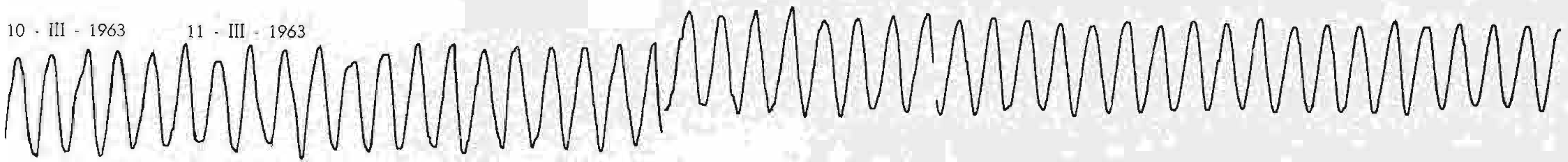
$17^{\mathrm{h}}$ $18^{\mathrm{h}}$ $19^{b}$

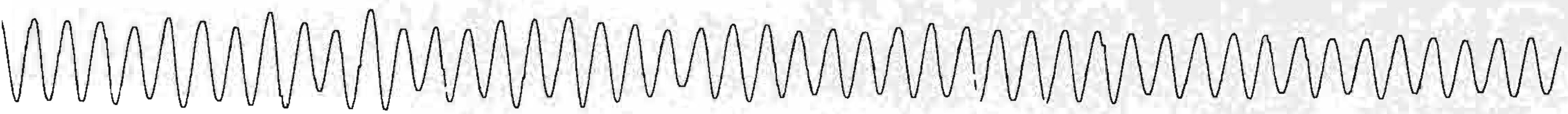
$20^{\text {h }}$ 21

$22^{\mathrm{h}}$

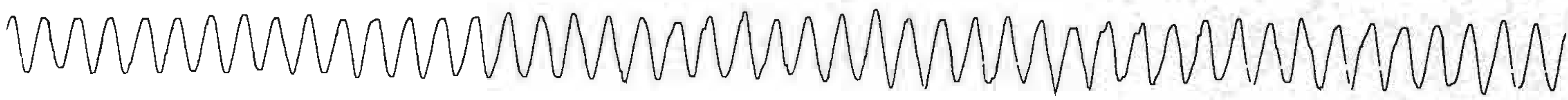
$23^{h}$ $24^{h}$ 01

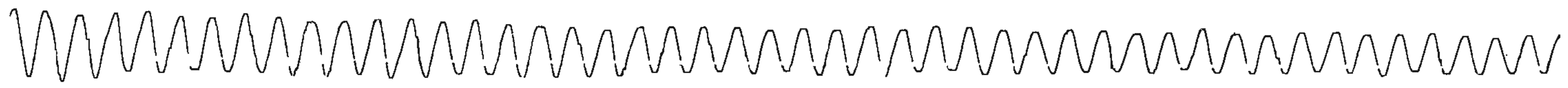
$02^{\mathrm{h}}$ 


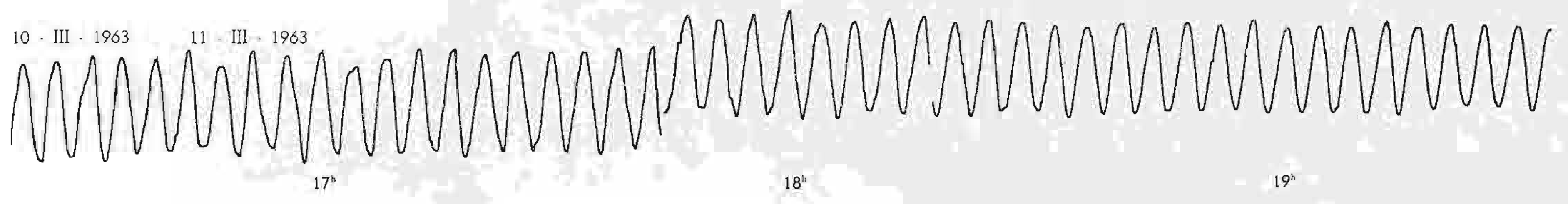
Fig. 13

\footnotetext{
basso

Fig. $13 a$

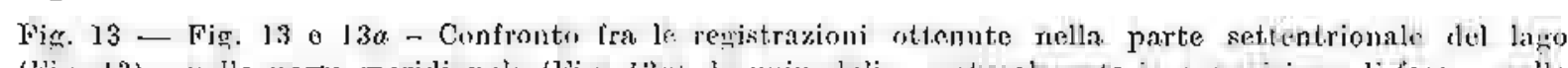

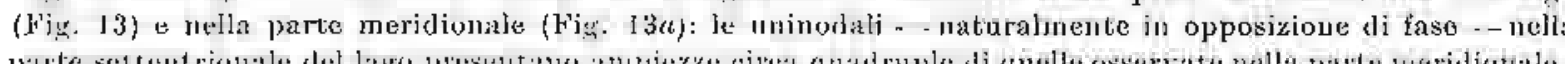

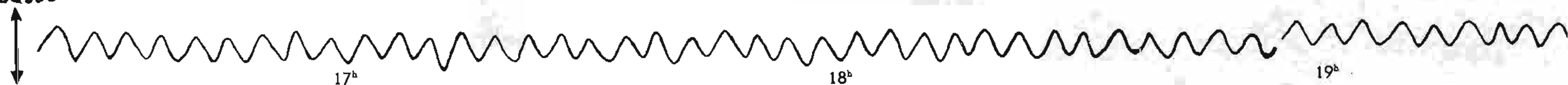


LAGO DI ALBANO

11 - III - 1963

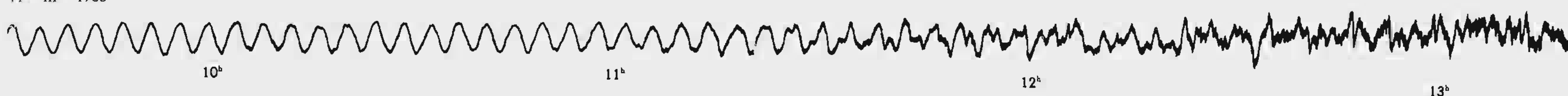

Fig. 14

$13^{b}$

11. III - 1963

$10^{\mathrm{h}}$

$11^{\mathrm{b}}$

$12^{\mathrm{h}}$

$13^{b}$

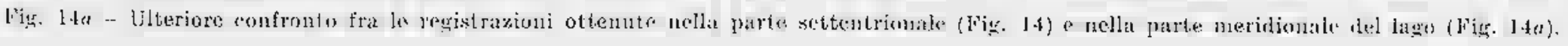




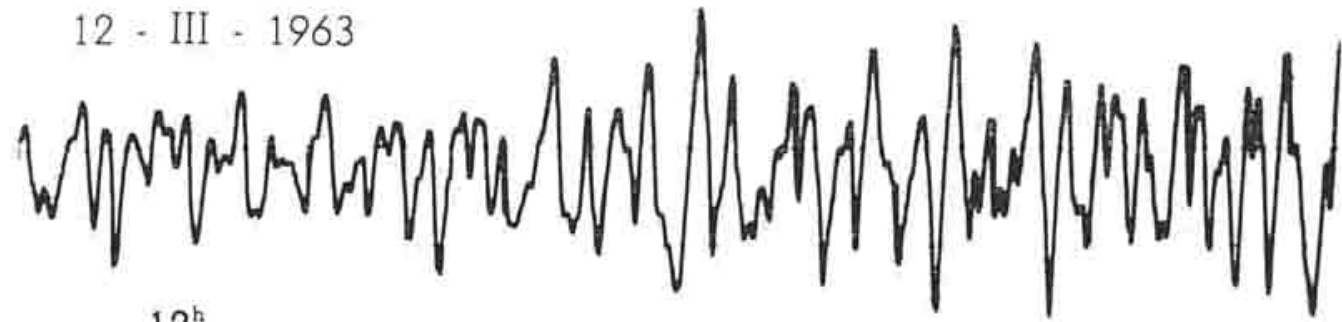
$12^{\mathrm{h}}$
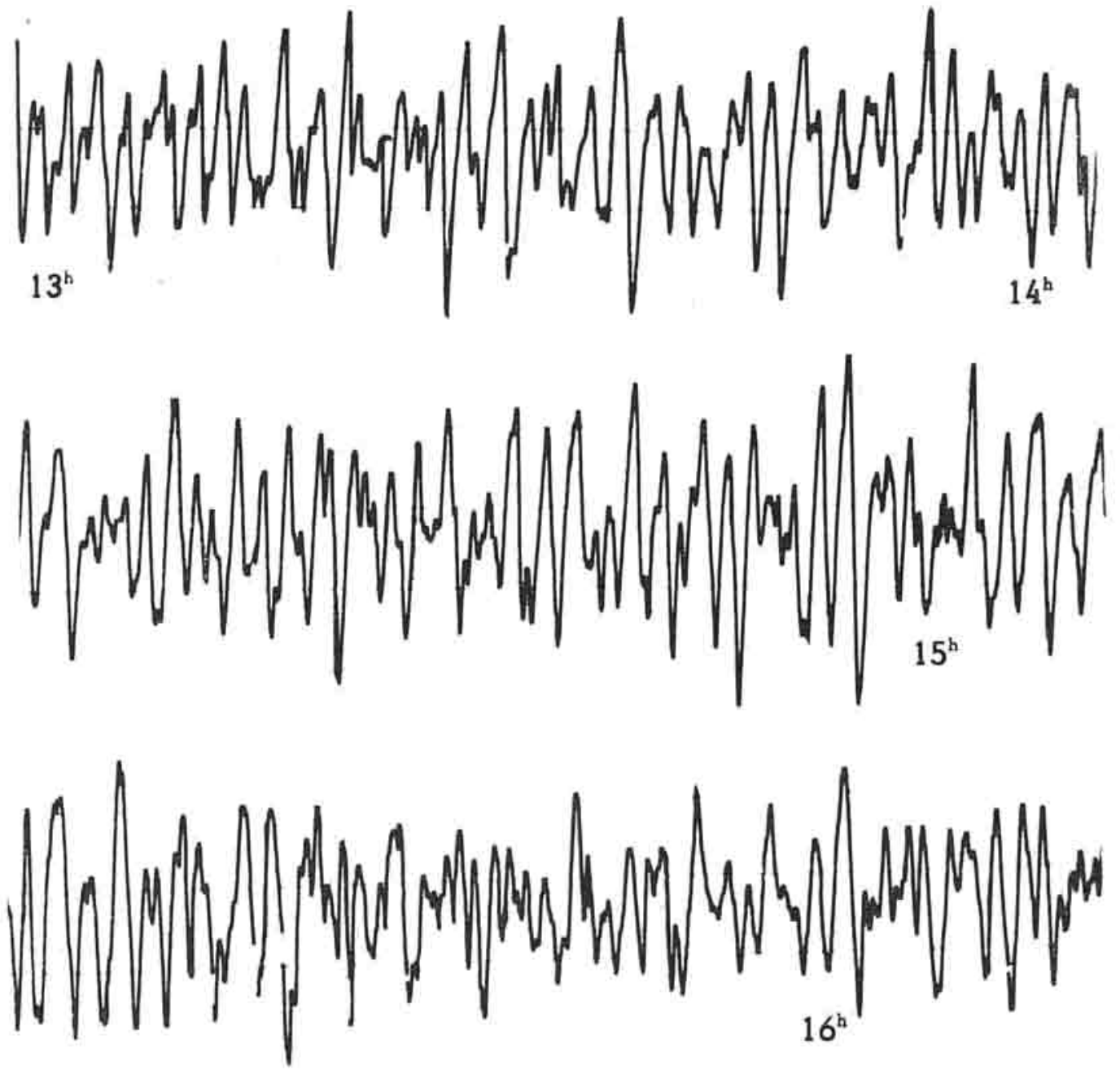

Fig. 15 - Uninodali e binodali registrate con ampiezze molto accutuate nella parte settentrionale del laigo. 